\title{
Программный модуль автоматизированного расчета параметров экранов защиты бортовой электронной аппаратуры от радиаиионного воздействия
}

\author{
1.A. Зинченко 1, д.m.н., npoфpeccop, lyudmillaa@mail.ru \\ B.B. Казаков 1, студент, kazakov.VADIM.2012@yandex.ru \\ A.A. Миронов ${ }^{1}$, студент, andrey_308@mail.ru \\ A.B. Дорофеев ${ }^{1}$, студент, alexanderzloizebroo@gmail.com \\ C.C. Кобъилкин 1, студент, kobylkinserh@gmail.com
}

${ }^{1}$ Московский государственный технический университет им. Н.Э. Баумана, кафедра "Проектирование и технология производства электронной аппаратуры", 2. Москва, 105005, Россия

При проектировании экранов защиты бортовой электронной аппаратуры от радиационного воздействия требуется проводить большое количество расчетов. Существующая методическая база для расчета таких экранов не позволяет выполнять автоматизированные анализ и обработку данных, полученных в результате расчетов, и проектировщику приходится делать это вручную.

Статья посвящена особенностям разработанного программного модуля, позволяющего автоматизировать процессы расчета параметров экранов защиты электронной аппаратуры от радиационного воздействия и влияния воздействия тяжелых заряженных частиц на экран радиационной защиты, а также осуществлять оценку стойкости различных проектных решений к воздействию тяжелых заряженных частиц.

Предложена клиент-серверная архитектура, с помощью которой можно организовать многопоточный расчет защитных экранов на множестве подключенных клиентов. Разработана база хранения уже рассчитанных параметров экранов с доступом онлайн. Детально рассмотрен принцип работы всей системы, а также представлены варианты сценариев ее использования. Разработан веб-интерфейс, позволяющий вносить данные для расчетов с любых устройств, имеющих доступ в Интернет, а также просматривать результаты уже проведенных расчетов.

Тестирование проводилось на серверах, где разработанный программный модуль показал стабильную работоспособность.

Ключевые слова: защитный экран, SRIM, клиент-серверная архитектура, wеb-интерфейс, заряженные частицы, автоматизация, распределенные вычисления.

Для решения различных задач космической программы РФ широко применяется электронная аппаратура. Использование микросхем обусловлено минимизацией веса устройств, проектируемых для космических приложений. Однако при проектировании бортовой электронной аппаратуры для работы в специфических условиях необходимо обеспечить ее защиту от радиационных воздействий [1-3].

При прохождении космического аппарата через пояс Ван Аллена бортовая электронная аппаратура подвергается воздействию тяжелых заряженных частии (ТЗЧ) [3], электронов и т.п. В результате микросхемы могут накапливать заряд, полученный от ТЗЧ [4-6]. В частности, под воздействием ТЗЧ могут меняться биты данных в памяти микросхемы, в результате чего возможны сбои и некорректная работа бортовой электронной аппаратуры. При этом наибольшей проникающей способностью в материал обладают протоны. В связи с этим возникает необходимость проектирования экранов радиационной защиты для обеспечения нужного уровня защиты бортовой электронной аппаратуры от протонов.

Допустимую толщину экрана для защиты от воздействия высокоэнергетичных ионов на различные материалы определяет комплекс программ SRIM (Stopping and Range of Ions in Matter) [7-9] или TRIM (Transport of Ions in Matter) [10-12]. Отметим, что программный комплекс SRIM не обновлялся с 2013 года, о чем свидетельствует дата последней версии [13]. В работе [14] эта методика была расширена для расчета параметров многослойных экранов радиационной защиты.

Одним из серьезных недостатков применения программы SRIM/TRIM в таких расчетах 
является сложность конфигурирования для выполнения расчета и анализа полученных данных, что требует значительных временных затрат инженера-проектировщика на исследование различных альтернативных проектных решений. Недостатком SRIM/TRIM является и необходимость ручного ввода: для расчета каждого альтернативного проектного решения требуется подготовить файл с входными параметрами или воспользоваться генератором входного файла. Оба метода используются для каждого расчета индивидуально, они нетривиальны и требуют определенных знаний по использованию программы SRIM/TRIM. В настоящее время это ПО доступно в двух версиях: TRIM для операционной системы DOS (также может работать в Windows в режиме эмуляции DOS) и SRIM для операционной системы Windows.

Необходимость разработки программного модуля автоматизации расчетов экранов радиационной защиты объясняется большим количеством альтернативных проектных решений, на проверку которых тратится много времени, при этом задача может быть формализуемой.

В данной статье описано разработанное ПО для автоматизации расчетов параметров многослойных экранов защиты электронной аппаратуры от воздействия ТЗЧ.

\section{Разработка и реализация программного модуля расчета параметров экранов защиты электронной аппаратуры от ТЗЧ}

Программный модуль предназначен для автоматизации расчета параметров многослойных экранов защиты электронной аппаратуры от воздействия ТЗЧ. Для решения поставленной задачи была проанализирована последовательность действий для получения искомых параметров. Для каждого материала, используемого в экране, необходимо подготовить входной файл расчета, запустить расчет и результаты его сохранить в файле. На последнем этапе нужно провести анализ полученных данных и сделать выводы о целесообразности использования того или иного проектного решения.

Каждый из перечисленных этапов работы можно реализовать в автоматическом режиме. На рисунке 1 представлен предложенный алгоритм работы пользователя с системой. На первом этапе пользователь вводит необходимые параметры для расчета: энергия протона, параметры мишени - толщина и материал. При

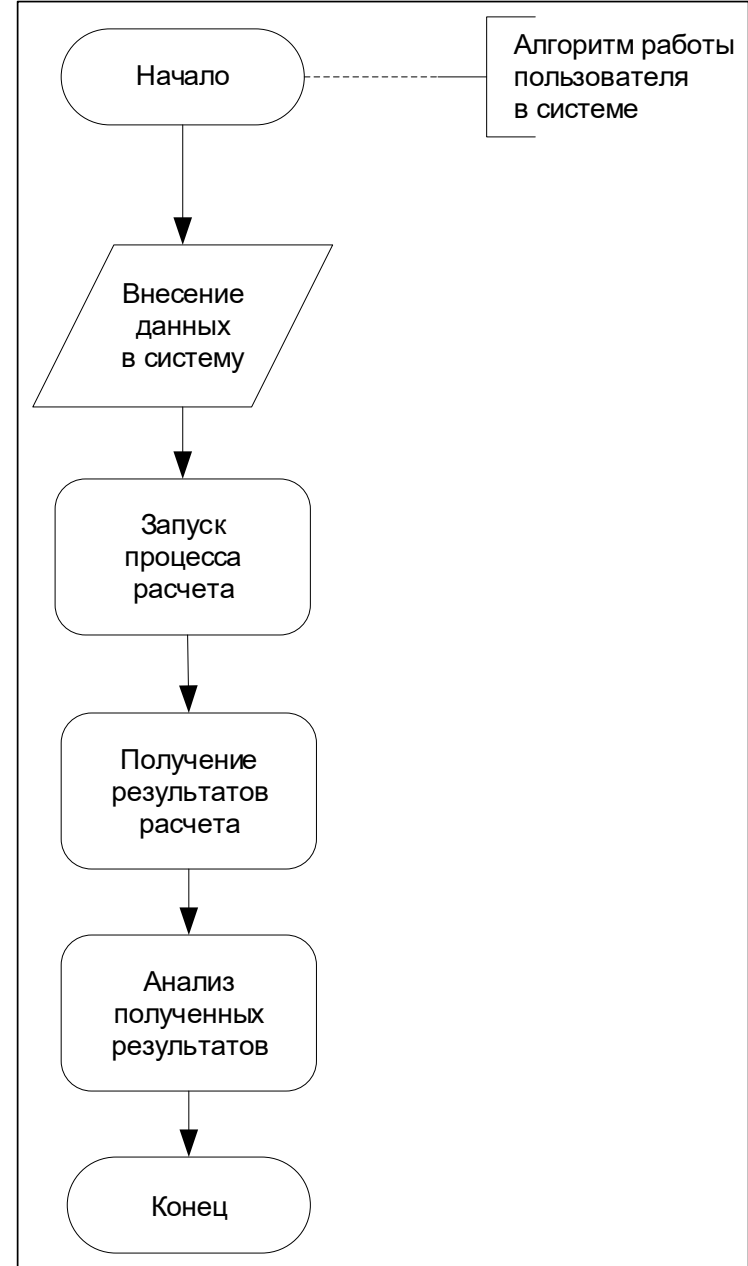

Рис. 1. Алгоритм работь пользователя в системе

Fig. 1. User work algorithm in the system

этом можно использовать уже имеющиеся в БД материалы или новые. После задания необходимых параметров исследователь может передать задачу на расчет, по окончании которого пользователь получает данные и может их проанализировать.

Для реализации распределенных вычислений была выбрана клиент-серверная архитектура (рис. 2).

При разделении исходной задачи на отдельные подзадачи на сервер были возложены следующие функции:

- хранение данных для расчета;

- хранение результатов расчета;

- управление расчетом множества альтернативных проектных решений;

- обработка результатов расчета (визуализация и анализ результата расчета одного проектного решения, а также сравнительный анализ различных проектных решений). 


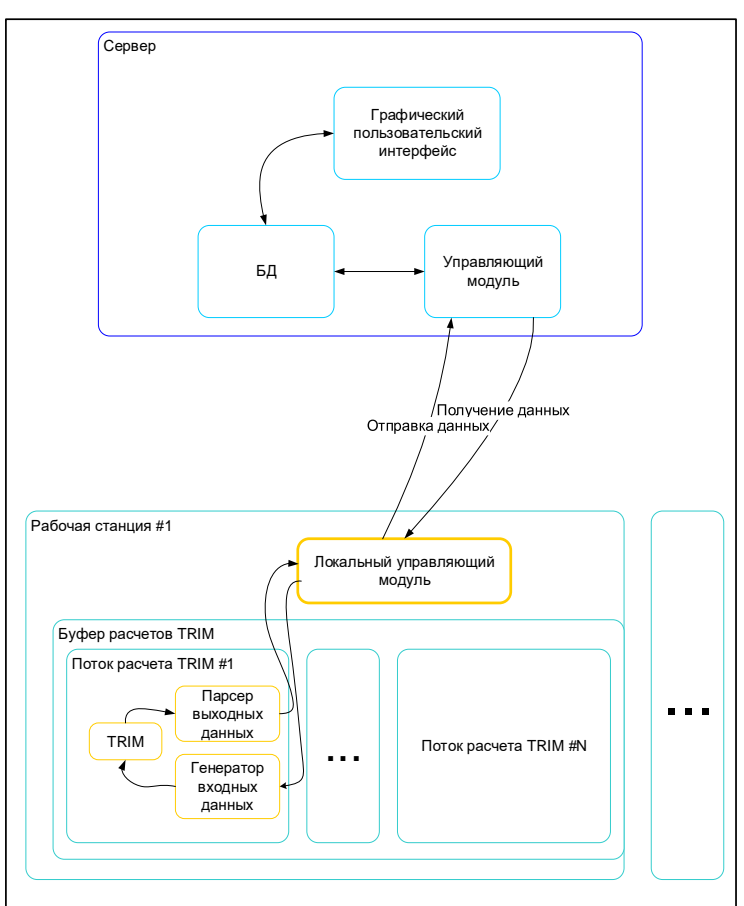

Рис. 2. Архитектура разработанной системь

Fig. 2. The developed system architecture

На клиента были возложены следующие задачи:

- получение данных для расчета из множества альтернативных проектных решений;

- генерация входного файла для расчета;

- расчет многослойных экранов с помощью программы SRIM/TRIM (далее SRIM);

- парсинг файлов результатов расчета;

- отправка данных на сервер.

Такой подход позволил реализовать распределенную систему вычислений.

На рисунке 3 представлен алгоритм работы подсистемы, отвечающей за управление очередью задач расчета. Основной целью данной подсистемы является распределение задач на клиентские машины, а также получение результатов расчетов.

На рисунке 4 приведен алгоритм работы подсистемы расчета. Основная цель данной подсистемы - получение очередной задачи для расчета и запуск моделирования. После окончания расчета подсистема отправляет результаты на сервер.

Клиент разделен на несколько программно независимых модулей (рис. 2). Основным является Локальный управляющий модуль. Он отвечает за получение данных о расчете, запуск расчета, управляет потоками расчета, отправляет данные на сервер. Модуль написан на языке программирования $\mathrm{C}++\mathrm{c}$ использова- нием фреймворка QT, что позволило обеспечить кроссплатформенность решения. Блок Генератор входных данных получает данные о расчете и на их основе генерирует входной файл для программы SRIM/TRIM. Генератор разработан на языке $\mathrm{C}++$ и является отдельным модулем. После окончания расчетов блок Парсер выходных данных обрабатывает выходные файлы SRIM и генерирует выходные данные.

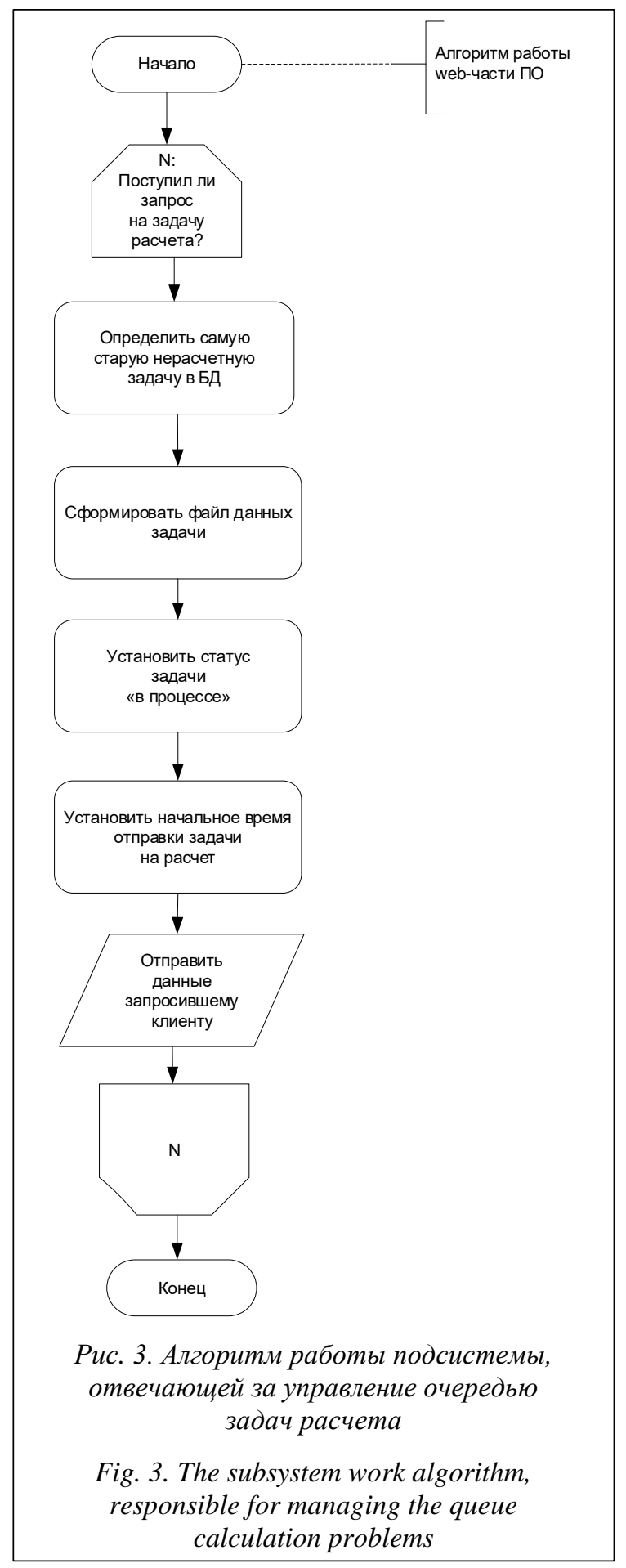




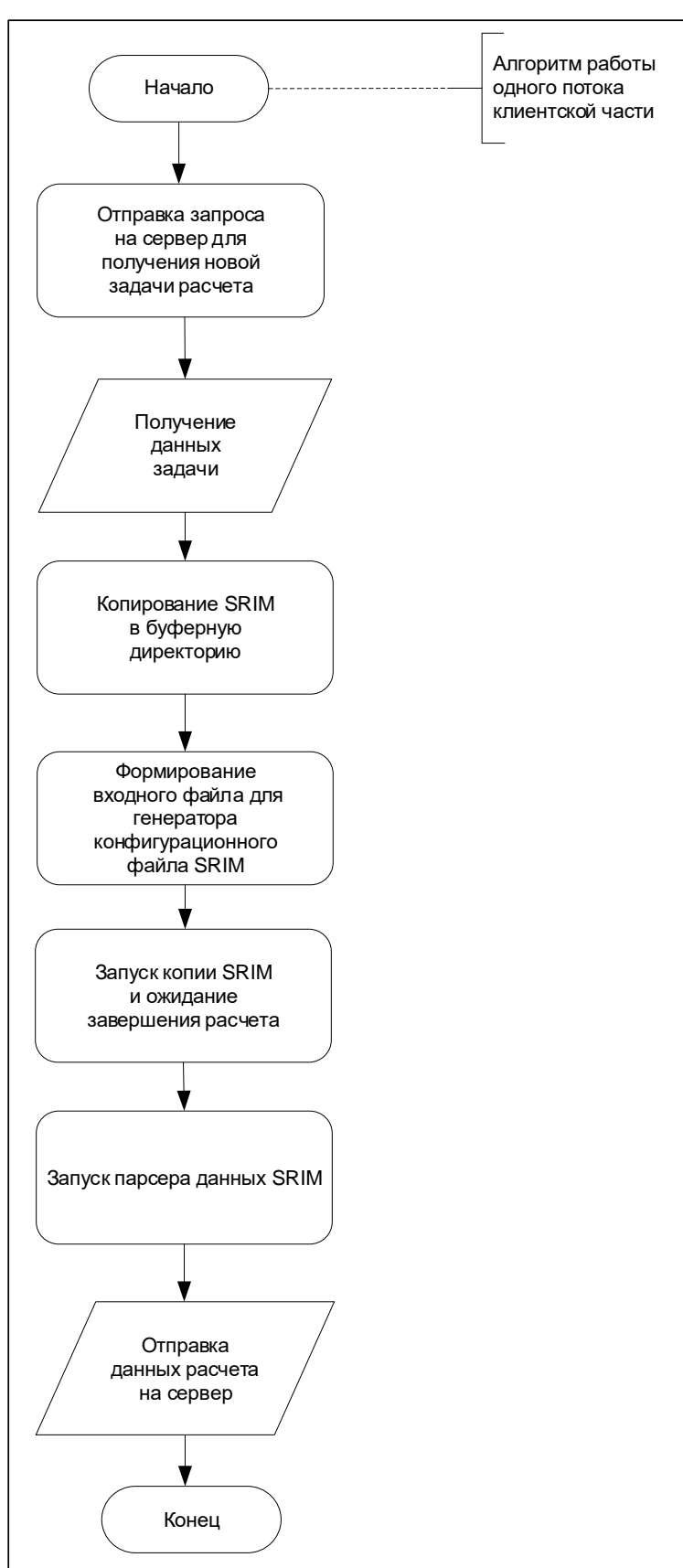

Рис. 4. Алгоритм работы модуля расчета

Fig. 4. Algorithm for the calculation module

Парсер написан на языке программирования Python. Такой выбор объясняется тем, что этот язык наиболее подходит для задач анализа текстовой информации и визуализации решений. Блок TRIM включает скопированные в буферную папку файлы программы SRIM.

Для реализации многопоточности контролирующий модуль программно создает новую папку для потока расчета, копирует туда SRIM, парсер и конвертер, генерирует файл для генератора по данным о последней задаче в множе- стве расчетов на сервере, запускает генератор После окончания работы генератора запускается SRIM, после окончания расчета запускается парсер, который, обработав полученный выходной файл, отправляет данные на сервер.

После выполнения всех операций на клиенте сервер получает данные о расчете и изменяет статус задачи в множестве расчета как рассчитанную. Также сервер сохраняет расчет, полученный от клиента.

Предложенный подход позволяет масштабировать ПО, запуская программу-клиент на большем числе компьютеров. Ограничением в выбранном подходе служит нагрузочная способность сервера, который отправляет данные на расчет и получает результаты.

Так как интерфейс добавления задач расположен на сервере, добавление задачи для расчета можно сделать с любого устройства с доступом в Интернет. Результаты расчета также можно просмотреть с любого устройства, имеющего доступ в Интернет, так как интерфейс анализа результатов располагается на сервере.

Блок Графический пользовательский интерфейс служит для добавления задач в множество расчетов и для анализа результатов расчета. Управляющий модуль содержит набор скриптов для получения и отправки данных на клиентские приложения для расчета. Блок БД хранит всю необходимую информацию для работы системы. Блок расчета TRIM \#1 представляет параллельно идущие процессы расчета, контролируемые Локальным управляющим модулем. Масштабировать систему можно, добавляя клиенты (персональные компьютеры) в кластер. Также есть возможность добавлять на каждом клиенте количество потоков для расчета.

Веб-интерфейс разработан с использованием фреймворка Bootstrap для разработки интерфейсов [15]. Преимуществами этого фреймворка являются кроссбраузерность, поддержка различных разрешений экранов и т.п.

Разработанный пользовательский интерфейс (см. http://www.swsys.ru/uploaded/image/ 2020-2/2020-2-dop/4.jpg) логически разделен на две части: страницу ввода данных об экранах и страницу вывода результатов расчета.

Слева отображается меню программного модуля. Основное окно отображает интерфейс для редактирования БД программного модуля. В разработанной системе все данные для расчета вводятся в специальные формы в соответствующие поля. Введенные данные в дальнейшем можно редактировать или удалять, а также 
использовать для последующих расчетов. При моделировании процесса проникновения ионов в материал экрана необходимо записать в соответствующую форму данные о материале с подробным описанием составляющего его сплава. Все данные можно просмотреть в соответствующем разделе на главной странице разработанного модуля. После внесения всех исходных данных необходимо поставить задачу в очередь на расчет. После окончания расчета результаты представляются на странице отчетов о выполнении расчетов в табличной форме. Также в выходных данных имеется информация о средней глубине проникновения ТЗЧ в исследуемый материал. Отметим, что такой информации в программе SRIM в явном виде нет, что затрудняет оценку альтернативных проектных решений.

Для тестирования работоспособности разработанного программного модуля было проведено сравнение результатов, полученных по методике [14], основанной на ручных операциях, и с использованием разработанного модуля. Клиентские машины представляли собой персональный компьютер следующей конфигурации: процессор - Intel Core i7-8565U, 4 ядра, 8 потоков; оперативная память - 16 Гб; графический ускоритель - NVIDIA GeForce MX150, Intel UHD Graphics 620.

Для тестирования работы программного модуля были заданы следующие параметры мишени (экрана защиты электронной аппаратуры от воздействия ТЗЧ): для слоя 1: материал алюминий, толщина - 2 мм; для слоя 2: материал - вольфрам, толщина - 1 мм.

Все слои располагались перпендикулярно относительно направления обстрела. В качестве падающей заряженной частицы были заданы ионы водорода с энергией 30 МэВ.

Как по ручной методике [14], так и с использованием разработанного программного модуля были получены одинаковые результаты расчета: средняя глубина проникновения ионов водорода с энергией 30 МэВ в многослойный экран защиты электронной аппаратуры - 2,58 мм, что свидетельствует о работоспособности программного модуля.

\section{Заключение}

В статье рассмотрены особенности реализации разработанного программного модуля автоматизации расчетов параметров многослойных экранов защиты электронной аппаратуры от воздействия тяжелых заряженных частиц. Выбранная клиент-серверная архитектура позволяет обеспечить удаленный доступ к параметрам уже рассчитанных экранов, а также расчет альтернативных проектных решений с использованием распределенных вычислений. Таким образом, реализованное ПО позволяет производить скоростную обработку информации и моделирование параметров экранов защиты в автоматизированном режиме.

Работа выполнена при частичной поддержке РФФИ, грант № 18-29-18043 мк.

\section{Лumepamypa}

1. Shakhnov V., Zinchenko L., Kosolapov I., Filippov I. Modeling and optimization of radiation tolerant microsystems. Proc. EMS'14, 2014, pp. 484-489.

2. Глушко А.А., Зинченко Л.А., Шахнов В.А. Моделирование воздействия тяжелых заряженных частиц на характеристики полевых транзисторов структуры «кремний на изоляторе» // Радиотехника и электроника. 2015. № 10. С. 1090-1096. DOI: 10.7868/s0033849415070074.

3. Кузнецов Н.В. Радиационная опасность на околоземных орбитах и межпланетных траекториях космических аппаратов. URL: http://nuclphys.sinp.msu.ru/crd/index.html (дата обращения: 15.11.2019).

4. Новиков Л.С., Милеев В.Н., Воронина Е.Н., Галанина Л.И., Маклецов А.А., Синолиц В.В. Радиационные воздействия на материалы космической техники // Поверхность. Рентгеновские, синхротронные и нейтронные исследования. 2009. № 3. С. 32-48.

5. Хаффнер Дж. Ядерное излучение и защита в космосе. М.: Атомиздат, 1971. 318 с.

6. Александров П.А., Жук В.И., Литвинов В.Л. Способы построения отказоустойчивых цифровых микросхем и оценки вероятности их отказа, вызванного облучением. М., 2019. 113 с.

7. Biersack J.P., Haggmark L. A Monte Carlo computer program for the transport of energetic ions in amorphous targets. Nuclear Instruments and Methods, 1980, vol. 174, pp. 257-269. DOI: 10.1016/0029$554 X(80) 90440-1$

8. Ziegler J.F., Biersack J.P., Littmark U. The stopping and range of ions in solids. NY, 1985, 321 p.

9. Ziegler J.F. SRIM-2003. Nuclear instruments and methods in physics research: Section B, 2004, vol. 219-220, pp. 1027-1036. DOI: 10.1016/j.nimb.2004.01.208. 
10. Galdikas A. Interaction of ions with condensed matter. NY, Nova Publ., 2000, 176 p.

11. Ziegler J.F. RBS/ERD simulation problems: Stopping powers, nuclear reactions and detector resolution. Nuclear instruments and methods in physics research: Section B, 1998, vol. 136-138, pp. 141-146. DOI: 10.1016/S0168-583X(97)00664-2.

12. Ziegler J.F., Ziegler M.D., Biersack J.P. SRIM - the stopping and range of ions in matter. 2010, vol. 268, iss. 11-12, pp. 1818-1823. DOI: 10.1016/j.nimb.2010.02.091.

13. SRIM-CODE. URL: http://www.ujfi.fei.stuba.sk/srim_support/ (дата обращения: 15.11.2019).

14. Шахнов В.А., Зинченко Л.А., Глушко А.А., Никитин И.В. Методика определения толщины экрана из многослойного материала для защиты микроэлектромеханических систем от воздействия протонов // XII Междунар. науч.-технич. конф. ПТСПИ: сб. матер. Суздаль, 2017. С. 91-93.

15. Bootstrap documentation. Framework for development web user interfaces. URL: https://getbootstrap. com/docs/4.4/getting-started/introduction/ (дата обращения: 15.11.2019).

\title{
Software module for automated calculation parameters for on-board electronic protection screens radiation protection equipment
}

\author{
L.A. Zinchenko ${ }^{1}$, Dr.Sc. (Engineering), Professor, lyudmillaa@mail.ru \\ V.V. Kazakov ${ }^{1}$, Master Student, kazakov.VADIM.2012@yandex.ru \\ A.A.Mironov ${ }^{1}$, Bachelor Student, andrey_308@mail.ru \\ A.V. Dorofeev ${ }^{1}$, Bachelor Student, alexanderzloizebroo@gmail.com \\ S.S. Kobylkin ${ }^{1}$, Bachelor Student, kobylkinserh@gmail.com
}

${ }^{1}$ Bauman Moscow State Technical University, Department "Design and Production Technology

of Electronic Equipment”, Moscow, 105005, Russian Federation

\begin{abstract}
When designing screens to protect onboard electronic equipment from radiation exposure, we need to perform a large number of calculations. The existing methodological base for calculating such screens does not allow performing automated analysis and processing of data obtained as a result of calculations, and the designer has to do this manually.

The article highlights the developed software module features that allow you to automate the calculating process for the electronic equipment safety shield parameters from radiation exposure.

The developed software module allows you to automate the process for calculating the effects of heavy charged particles on the radiation protection screen and evaluate the resistance of various design solutions to the effects of heavy charged particles.

The article offers a client-server architecture that can be used to organize the multithreaded calculation of safety shields on multiple connected clients. There is a database for storing already calculated screen parameters with online access. There is a principle of operation of the entire system in detail, as well as options for use cases. The authors have developed a web interface that allows you to enter data for calculations from any devices that have Internet access, as well as view the calculation results that have already been performed.

Testing was on servers where the developed software module showed stable performance.

Keywords: shield, SRIM, client-server architecture, WEB interface, charged particles, automation, distributed calculations.
\end{abstract}

Acknowledgements. This work was with partial support by RFBR, project no. 18-29-18043 мк.

\section{References}

1. Shakhnov V., Zinchenko L., Kosolapov I., Filippov I. Modeling and optimization of radiation tolerant microsystems. Proc. EMS '14, 2014, pp. 484-489.

2. Glushko A.A., Zinchenko L.A., Shakhnov V.A. Modeling of influence of heavy charged particles on characteristics of field transistors of structure "silicon on insulator". Radio Engineering and Electronics, 2015, no. 10, pp. 1090-1096. DOI: 10.7868/s0033849415070074 (in Russ.). 
3. Kuznetsov N.V. Radiation Danger in Near-Earth Orbits and Interplanetary Trajectories of Spacecraft. Available at: http://nuclphys.sinp.msu.ru/crd/index.html (accessed November 15, 2019).

4. Novikov L.S., Mileev V.N., Voronina E.N., Galanina L.I., Makletsov A.A., Sinolic V.V. Radiation effects on materials in space technology. Surface. X-ray, Synchrotron and Neutron Studies, 2009, no. 3, pp. $32-48$ (in Russ.).

5. Khaffner Dzh. Nuclear Radiation and Protection in Space (Russ. ed.: Moscow, 1971, 318 p.).

6. Alexandrov P.A., Zhuk V.I., Litvinov V.L. Methods of Constructing Fault-Tolerant Digital Chips and Assessing the Probability of their Failure Caused by Radiation. Moscow, 2019, 113 p. (in Russ.).

7. Biersack J.P., Haggmark L. A Monte Carlo computer program for the transport of energetic ions in amorphous targets. Nuclear Instruments and Methods, 1980, vol. 174, pp. 257-269. DOI: 10.1016/0029$554 \mathrm{X}(80) 90440-1$.

8. Ziegler J.F., Biersack J.P., Littmark U. The Stopping and Range of Ions in Solids, NY, 1985, 321 p.

9. Ziegler J.F. SRIM-2003. Nuclear Instruments and Methods in Physics Research: Section B, 2004, vol. 219-220, pp. 1027-1036. DOI: 10.1016/j.nimb.2004.01.208.

10. Galdikas A. Interaction of Ions with Condensed Matter. NY, Nova Publ., 2000, 176 p.

11. Ziegler J.F. RBS/ERD simulation problems: Stopping powers, nuclear reactions and detector resolution. Nuclear Instruments and Methods in Physics Research: Section B, 1998, vol. 136-138, pp. 141-146. DOI: 10.1016/S0168-583X(97)00664-2.

12.Ziegler J.F., Ziegler M.D., Biersack J.P. SRIM - the Stopping and Range of Ions in Matter. 2010, vol. 268, iss. 11-12, pp. 1818-1823. DOI: 10.1016/j.nimb.2010.02.091.

13. SRIM-CODE. Available at: http://www.ujfi.fei.stuba.sk/srim_support/ (accessed November 15, 2019).

14. Shakhnov V.A., Zinchenko L.A., Glushko A.A., Nikitin I.V. Method for determining the thickness of a screen made of a multilayer material to protect microelectromechanical systems from the effects of protons. Proc. Conf. PTMM, Suzdal, 2017, pp. 91-93 (in Russ.).

15. Bootstrap Documentation. Framework for Development Web User Interfaces. Available at: https://getbootstrap.com/docs/4.4/getting-started/introduction/ (accessed November 15, 2019).

\section{Для цитирования}

Зинченко А.А., Казаков В.В., Миронов А.А., Дорофеев А.В., Кобылкин С.С. Программный модуль автоматизированного расчета параметров экранов защиты бортовой эмектронной аппаратуры от радиационного воздействия // Программные продукты и системы. 2020. Т. 33. № 2. C. 236-242. DOI: $10.15827 / 0236-235 X .130 .236-242$.

\section{For citation}

Zinchenko L.A., Kazakov V.V., Mironov A.A., Dorofeev A.V., Kobylkin S.S. Software module for automated calculation parameters for on-board electronic protection screens radiation protection equipment. Software \& Systems, 2020, vol. 33, no. 2, pp. 236-242 (in Russ.). DOI: 10.15827/ 0236-235X.130.236-242. 